\title{
On the use of graphs within constraint-programming
}

\author{
Jean-Guillaume Fages ${ }^{1}$
}

Published online: 16 September 2015

(C) Springer Science+Business Media New York 2015

\begin{abstract}
Many IT applications require solving decision problems that are hard from a mathematical point of view. Constraint-programming enables to model and solve some of these problems. Among them, some are defined over graphs. Beyond the difficulty stemming from each of these problems, the size of the instance to solve increases the difficulty of the task. This $\mathrm{PhD}$ thesis is about the use of graphs within constraint-programming, in order to improve its scalability. First, we study the use of constraint-programming to solve some graph problems involving the computation of trees and Hamiltonian paths and cycles. These problems are important and can be found in many industrial applications. Both filtering and search are investigated. Next, we move on problems that are no longer defined in terms of graph properties. We then study the use of graphs to propagate global constraints. In particular, we suggest a generic schema, relying on a graph structure, to dynamically decompose filtering algorithms. The central theme in this work is the use of graph concepts at the origin of every reasoning and the practical will to increase the size of problems that can be addressed in constraint-programming.
\end{abstract}

School: Ecole des Mines de Nantes, LINA, CNRS, INRIA, France

\section{Supervisors:}

Xavier Lorca

Nicolas Beldiceanu

Graduated: Thursday, October 23, 2014

Link to full text: http://www.a4cp.org/sites/default/files/jean-guillaume_fages_-_on_the_use_ of_graphs_within_constraint-programming.pdf

Jean-Guillaume Fages

jg.fages@cosling.com; http://www.cosling.com

1 COSLING S.A.S., 2 Rue Alfred Kastler, 44300 Nantes, France 


\section{Notes}

- Co-winner of the ACP doctoral research award 2015

- Best French PhD thesis in Artificial Intelligence (AFIA PhD award 2015) 